\title{
A robust multimedia surveillance system for people counting
}

Zeyad Q. H. Al-Zaydi' ${ }^{1}$, David L. Ndzi' ${ }^{1}$, Munirah L. Kamarudin ${ }^{2}$, Ammar Zakaria $^{3}$, Ali Y.M. Shakaff ${ }^{3}$

${ }^{1}$ School of Engineering, University of Portsmouth, Portsmouth, PO1 3DJ, UK

${ }^{2}$ School of Computer and Communication Engineering, University Malaysia Perlis, Perlis, Malaysia

${ }^{3}$ School of Mechatronic Engineering, University Malaysia Perlis, Perlis, Malaysia Tel: $+44(0) 2392842551$

E-mails: \{zeyad.al-zaydi, david.ndzi\}@ port.ac.uk, \{latifahmunirah, ammarzakaria, aliyeon\}@unimap.edu.my

\section{Highlight of the Paper}

- Two people counting algorithms based on CCTV cameras are proposed.

- Training error, set-up time and cost have been reduced by the proposed system.

- Motion edges, grid map, pixel-wise and fusion techniques are used in the proposed algorithms.

- Two indoor and outdoor datasets are used for evaluation.

- The accuracy of the proposed system is, at least, comparable with the state of the art methods. 


\title{
A robust multimedia surveillance system for people counting
}

\author{
Zeyad Q. H. Al-Zaydi', David L. Ndzi' ${ }^{1}$, Munirah L. Kamarudin², Ammar Zakariaª , Ali Y.M. Shakaff ${ }^{3}$ \\ ${ }^{1}$ School of Engineering, University of Portsmouth, Portsmouth, PO1 3DJ, UK \\ ${ }^{2}$ School of Computer and Communication Engineering, University Malaysia Perlis, Perlis, Malaysia \\ ${ }^{3}$ School of Mechatronic Engineering, University Malaysia Perlis, Perlis, Malaysia \\ Tel: +44(0)239284 2551 \\ E-mails: \{zeyad.al-zaydi, david.ndzi\}@ port.ac.uk, \{latifahmunirah, ammarzakaria, aliyeon\}@unimap.edu.my
}

\begin{abstract}
Closed circuit television cameras (CCTV) are widely used in monitoring. This paper presents an intelligent CCTV crowd counting system based on two algorithms that estimate the density of each pixel in each frame and use it as a basis for counting people. One algorithm uses scale-invariant feature transform (SIFT) features and clustering to represent pixels of frames (SIFT algorithm) and the other uses features from accelerated segment test (FAST) corner points with SIFT features (SIFT-FAST algorithm). Each algorithm is designed using a novel combination of pixel-wise, motion-region, grid map, background segmentation using Gaussian mixture model (GMM) and edge detection. A fusion technique is proposed and used to validate the accuracy by combining the result of the algorithms at frame level. The proposed system is more practical than the state of the art regression methods because it is trained with a small number of frames so it is relatively easy to deploy. In addition, it reduces the training error, set-up time, cost and open the door to develop more accurate people detection methods. The University of California (UCSD) and Mall datasets have been used to test the proposed algorithms. The mean deviation error, mean squared error and the mean absolute error of the proposed system are less than 0.1, 16.5 and 3.1, respectively, for the Mall dataset and less than 0.07, 5.5 and 1.9, respectively, for UCSD dataset.
\end{abstract}

Keywords: Crowd counting systems, monitoring, CCTV cameras, background segmentation.

\section{INTRODUCTION}

Closed circuit television cameras (CCTV) have already become ubiquitous and their use is growing exponentially. For instance, 4.2 million CCTV cameras were used in the United Kingdom [1] in 2004 and an estimated up to around 5.9 million in 2015 [2]. People counting systems are one of the most 
challenging systems in computer vision to implement [3]-[9]. People counting is a useful task for safety, security and operational purposes and can be important for improving awareness [7], [8], [10][12]. The number of people in a given space can be used to develop business intelligence, such as improving location of products within a shop and finding the number of visitors [10]-[12]. Crowd management [11], transport [13] and staff planning applications can be improved by using this kind of information. Heating, lighting and air conditioning can also be optimised using people counting and distribution information to enhance energy management [14], [15], or to improve emergency evacuation plan [15].

A significant amount of research has been carried out to find an accurate computer vision solution but there are still many challenges that need to be resolved. These include occlusions, varying lighting, long processing time and improving the accuracy in image processing [4], [6], [8], [16].

This work distinguishes itself with the following four main contributions. First, a new combination of SIFT and FAST features with pixel-wise technique is used to improve the accuracy. Second, motion edge pixels are used instead of foreground pixels to reduce the number of SIFT descriptors required. Third, a combination of grid map and pixel-wise technique is used to improve the cluster classification in frames which enables similar clusters in different cells to be assigned different densities depending on their location in the frame. Fourth, the algorithms are comprehensively tested and validated using two datasets, the University of California, San Diego (UCSD) and Mall datasets [17], [18].

\section{RELATED WORK}

Crowd counting can be classified into four categories; crowd counting based on detection, clustering, regression and optimisation.

\subsection{People detection based algorithms}

Detection based algorithms start by detecting people individually and then counting them [16]. The detection process depends either on the person's entire body or parts of the body such as face, head or head-shoulder [16]. The main advantages of these algorithms are that they count people and find their locations as well, therefore they are useful in people tracking [5]. The main disadvantages of these algorithms are that they are severely affected by varying lighting, occlusion and have long processing times [19]. They achieve good results in sparsely populated scenarios, whereas in crowded scenarios, the accuracy decreases significantly [5]. In addition, a high resolution camera is required to obtain a good accuracy [5]. Triggs and Dalal proposed histogram of oriented gradients (HOG) method thereby creating a basis for the development of a fast appearance-based detection algorithm [20]. Many improvements of the HOG technique have been proposed. One of the most promising variants is the 
fastest pedestrian detection in the west (FPDW) which has significantly increased the speed of detection [21].

Pedestrian detection is constrained to horizontal or vertical camera angles. In people counting, horizontal camera angles can be used but a vertical or downward facing angle is often preferred to minimise occlusions [22]. A majority of commercial people counting products are cameras that are placed on the ceiling pointing downwards to get the best view. However, this is not an optimal set-up if the detection area needs to be maximized.

People detection based algorithms can be classified into six categories: full body detection [20], [23], [24]; part body detection [25]-[27]; 3D camera detection [28]; shape matching detection, where ellipse and Bernoulli shapes are used to identify and count people in each blob [29], [30]; multi-camera detection, which is used to avoid occlusion [31]; and density-aware detection, which is used to reduce the false positive per image (FPPI) in low crowd density locations and decreases the miss rate in high crowd density locations in the frames [32].

\subsection{Features trajectories clustering based algorithms}

Clustering based algorithms track visual features over time and the feature trajectories are then clustered into unique tracks using temporal, spatial and other factors [33]-[36]. The number of clusters is the estimated number of people [37]. Different approaches have been used to study the similarities between trajectories such as Dynamic Time Warping (DTW). DTW is a time series method that is widely used to measure similarities between two temporal sequences [38], [39]. Kanade-LucasTomasi (KLT) feature-matching algorithm is sometimes used to find the trajectories of features [34]. The advantages of clustering based algorithms are that they can decrease the occlusion and the angle of the camera effects [40]. However, their accuracies significantly decrease in highly crowded environments with cluttered background and heavy occlusion. A complicated trajectory management technique is required to assess the similarities of trajectories with different lengths, which is another limitation of these algorithms [41]. In addition, errors in the number of people due to the cohesiveness of features that belong to different people also affect their accuracies [41]. These algorithms also require high video frame rate to work well because motion information can reliably be extracted [18]. Features trajectory clustering algorithms can be used to count people but it is difficult to use them in a real-time environments due to their long processing times.

\subsection{Low-level features regression based algorithms}

Regression based algorithms usually consist of three steps, starting with a background segmentation that is used on a frame by frame basis to detect the foreground information. Low-level features are then extracted from the foreground such as edge features [18], [42]-[45], segment features [5], [17], [42], [44]-[49], texture features [45], [51], [80], [81] and keypoints [84]. A regression function is 
then trained using these features to find the relationship between the number of people and the extracted features which is then used to estimate the number of people [35]. Various types of regression functions have been used such as support vector machine tree [50], [51], linear [52], [53], neural networks [42]-[45] and Gaussian process algorithms [17], [37], [47]-[49]. A significant amount of research has been carried out to improve these algorithms by varying the number of features. Some other researchers have tried to improve them by using more than one regression function and then choose the best fitting features [54]. The main advantages of these algorithms are that the accuracy is higher than feature trajectory clustering and detection based algorithm in crowded scenarios, and the computational time is shorter [18], [19], [54]. Their main disadvantage is that different training datasets are required with different environments or camera set-ups [35]. Some new contributions have also been presented to improve their accuracies, handling occlusions and adapt to new environments. Recent technique in crowd counting has been tested using static pictures from crowded environments [55]. A deep-learning approach that uses convolutional neural networks to predict the number of people has been proposed in that technique. Occlussion is another problem that a new proposed technique tries to minimise [56]. Research in [56] takes occlusion into account by using two regression functions, one for the low occlusion frames and the second for the high occlusion frames. In addition, adaptive combination of features is used in each environment according to their nature. Statistical features have been used by Hafeezallah et al [57] to train a neural network to develope a highly accurate crowd counting algorithm. The differences of the sequential frames with curvelet transform has been proposed by [57] to improve the accuracy. A random projection forest, as a regression function, has also been proposed by other researchres to increase the maximum number of features that is used for training [58]. A small number of features can be handled by traditional regression functions which can negatively affect the performances of crowd counting systems. Aravinda et al [59] have proposed a combination of optical flow for motion cues and hierarchical clustering to estimate the crowd density. Hierarchical clustering have been in [59] used to isolate distinct pixels that correspond to different people in the frame. Multi-cameras knowledge transfer technique has been used by Nick et al [60] to provide different views of the crowd which are used to minimise occlusion and improve performance. The main disadvantage of the technique is the long set-up time required and the high cost of the hardware. Finally, a quadratic programming technique is used with a regression function and network flow constraints to improve the accuracy of estimating the number of people [61]. They take into account the temporal domain of a series of frames to improve the accuracy. Regression based algorithms are classified into three categories; holistic, histograms (intermediate) and local algorithms [62].

Holistic algorithms use global image features and one regression function for the whole frame [17], [42], [44], [45], [47]-[49]. The types of features that used by these algorithms include foreground, 
edge, keypoints and texture features. A limitation of these algorithms is that they apply one global regression function over the whole image thereby not taking into account the high variability of crowd distribution, behaviour and density in different regions of the image [62].

Histogram features are used by histograms algorithms such as, edge orientation histogram, blob size histogram and histogram of oriented gradients (HOG) [63], [64]. One global regression function is trained by these features to find the estimated number of people. These algorithms use histogram bin magnitude and edge direction to avoid noise and to distinguish people, respectively. Histograms algorithms also ignore high variations in crowd behaviour, distribution and density in different regions of the image [62].

Local algorithms count the number of people by partitioning the frame into several regions and one local regression function is trained for each region to count the total number of people in the whole frame. The regions can be cells having regular or irregular sizes [18] or the regions can be foreground blobs and the total number of people is counted by summing the numbers in all regions [43], [65][68].

\subsection{Pixel-wise optimisation based algorithms}

Some researchers use pixel-wise techniques to estimate the number of people [69]. In this approach, the density of each pixel is found and then integrated over the whole frame to estimate the total number of people [69]. Optimisation is used instead of regression to train crowd counting systems. This approach can be used to improve people detection algorithms by combining it with full or part body detection based algorithms [32]. Full body, head and head-shoulder detection based algorithms can be improved and the accuracy can be increased by using the density of pixels [32]. The aim of this combination is to reduce the false positive per image (FPPI) in low crowd density locations in the frames which happens when it inaccurately detects the presence of people when there is actually nobody. In addition, this approach decreases the miss rate in high crowd density locations in the frames. Pixel-wise optimisation based algorithms can be trained using a small number of frames in comparison to regression based algorithms [69]. As a consequence, the set-up time of the system can be reduced by more than $25 \%$ in comparison to regression based algorithms which lead also to low set-up cost. Using a large number of training frames can negatively affect the accuracy of the training because manually annotation is an error-prone task.

\section{SYSTEM DESIGN}

In this paper, the proposed system depends on supervised learning to estimate the number of people. The training frames are annotated and Gaussian representation is used to represent people. Quadratic programming is used for learning and maximum excess over subarrays distance $\left(D_{\text {MESA }}\right)$ is used to 
measure the difference between the true and predicted count which represent the loss function as given by equation (2).

The proposed system assumes that each pixel (p) in a frame is represented by a SIFT or SIFT-FAST feature vector. The density function of each pixel is represented as a linear transformation of the pixel representation $\left(\mathrm{x}_{\mathrm{p}}\right)$ as given by equation (1);

$$
F(p)=\mathrm{w}^{\mathrm{T}} \mathrm{x}_{\mathrm{p}}
$$

Where $\mathrm{w}^{\mathrm{T}}$ is the weight of each pixel in the frame. At the learning stage, a training frames set with their ground truth (true count) are used to find the correct weight $\left(\mathrm{w}^{\mathrm{T}}\right)$ of each pixel. Then the densities of all pixels in the frame are summed to find the predicted count. $\mathrm{D}_{\mathrm{MESA}}$ is used to compare between the predicted count and true count as a loss function. $D_{\text {MESA }}$ is defined as [32];

$$
D_{M E S A}(F 1, F 2)=\max \left|\sum_{p \in B} F 1(p)-\sum_{p \in B} F 2(p)\right|
$$

Where $F 1(p)$ and $F 2(p)$ are the predicted count and true count of people in a frame. $\mathrm{D}_{\mathrm{MESA}}$ is chosen for the proposed system because it is not significantly affected by jitter and noise but it has a strong relationship with the number and positions of people [69]. The ultimate goal of the learning stage is to find the best weight for each pixel that minimises the sum of the errors between the true counts and the predicted counts (the loss function) [69];

$$
w=\operatorname{argmin}_{w}\left(w^{T} w+\gamma \sum_{i=1}^{N} D_{M E S A}\right)
$$

Where $\gamma$ is a scalar parameter to control the regularization strength, $\operatorname{argmin}_{w}$ represents the best weight that minimises the $D_{M E S A}$. Quadratic programming can be used to solve equation (3) by using;

$$
\min _{w, \xi_{1}, \ldots \ldots, \xi_{N}}\left(w^{T} w+\gamma \sum_{i=1}^{N} \xi_{i}\right)
$$

Subject to;

$$
\xi_{i} \geq \sum_{p \in B}(F 1(p)-F 2(p)), \quad \xi_{i} \geq \sum_{p \in B}(F 2(p)-F 1(p))
$$

Where $\xi_{i}$ are the auxiliary variables of training frames. Quadratic programming uses iterations to optimise the results and find the best weight $\left(\mathrm{w}^{\mathrm{T}}\right)$ of each pixel. The iterations terminate when the right side of equation (5) is within $\left(\xi_{i}+\beta\right)$ factor. $\beta$ is a small constant $(\beta<<1)$. It uses to decrease the number of iterations and faster convergence. Choosing $\beta$ equal to 0 solves the equations (4) and (5) exactly. However, the convergence will finish faster if $\beta$ is chosen to a very small value and that will not affect the performance of training [69]. In the experiments of the proposed system, $\beta$ has been chosen to be equal to 0.001 . The flow diagram of the proposed system is illustrated in the Figure 1. It consists of two counting algorithms, one video source and one fusion model. 


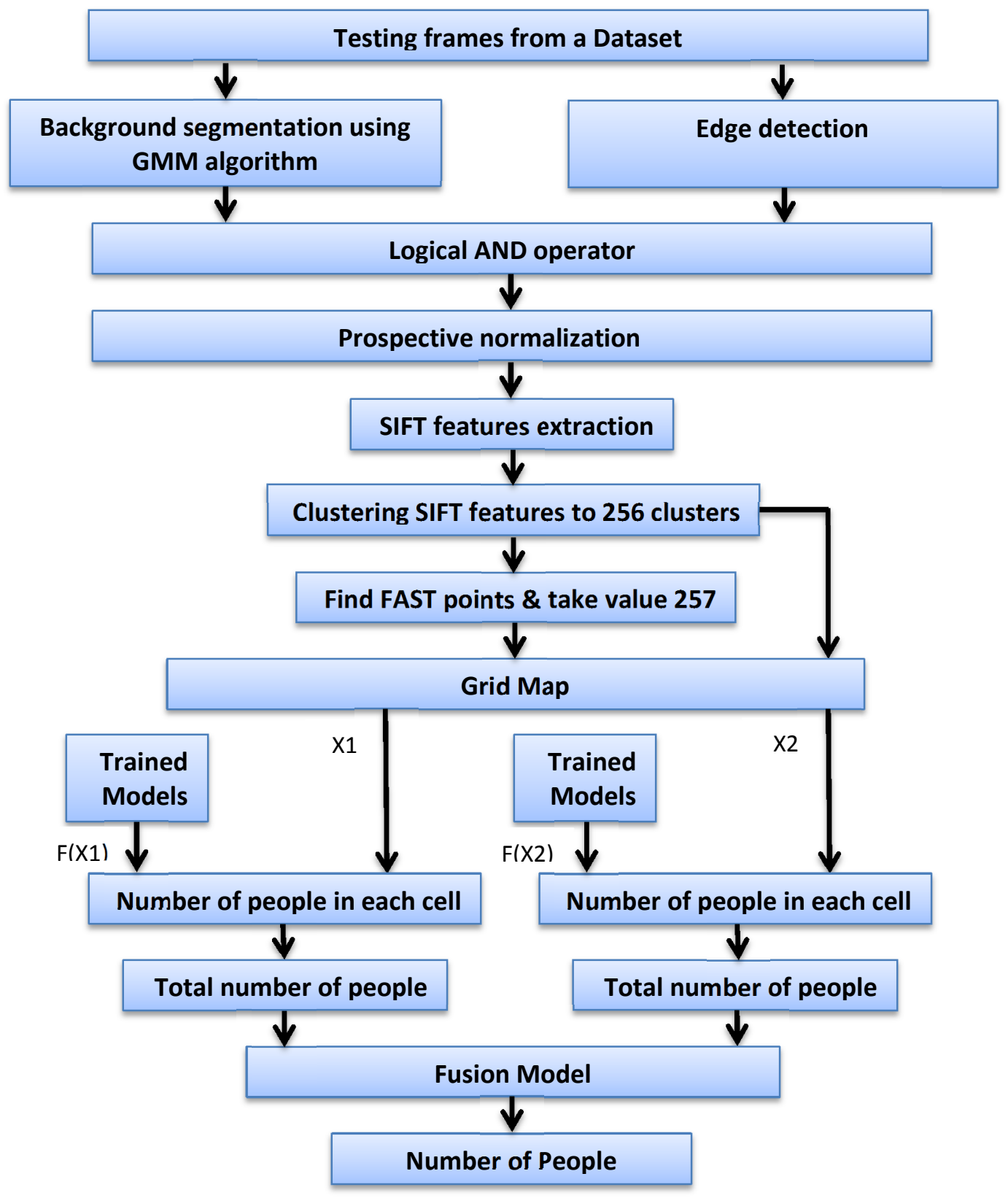

Figure 1: Flow diagram of the proposed system.

\subsection{Algorithm 1: SIFT features algorithm}

This algorithm combines the following techniques to count the number of people; motion edges, SIFT descriptors, gird map and pixel-wise techniques. This combination that is used to find the density of 
each pixel, is novel. Edge pixels are used because their number is less than foreground pixels. As a consequence, the required time to find the SIFT descriptors and cluster them in a frame will be significantly reduced which makes the proposed system faster than other people counting techniques based on $\mathrm{D}_{\mathrm{MESA}}$ optimisation. There is a high correlation between SIFT descriptors and the number of people. This is difficult for quadratic programming to be used to find the density for a large number of SIFT descriptors (equal to the number of edge motion pixels). To solve this problem, clustering is used to reduce the number of SIFT descriptors to 256 clusters. The main disadvantage of using clustering is that many SIFT descriptors can be grouped into one cluster to reduce the problem space but they represent different densities. Grid map is used to improve the cluster classification in the frames which enables similar clusters in different cells to be assigned different densities depending on their location in the frame. The proposed algorithm can better adapt to high variations in crowd behaviours, distributions and densities. As a result, the accuracy is improved. Figure 2 shows the flow diagram of this algorithm. The procedure of the algorithm is illustrated in the following steps;

1- Implement Gaussian mixture model (GMM) to find the foreground information of the frame.

$F_{G M M}=\operatorname{GMM}(i, j)$

Where $F_{G M M}$ is the foreground pixels of the frame and $G M M(i, j)$ is the Gaussian mixture model of each pixel of the frame.

2- Implement edge detection to find the edges of the frame.

$F_{E d g e}=E(i, j)$

Where $F_{E d g e}$ is the edge of the frame and $E(i, j)$ is the detected edge of each pixel of the frame.

3- Perform logical (AND) operation between the foreground pixels of the frame and the detected edge to find the motion edge of the frame.

$F_{\text {motion edge }}=F_{G M M}(i, j) \& \& F_{\text {Edge }}(i, j)$

Where $F_{\text {motion edge }}$ is the motion edge for the frame.

4- The pixels in each line of the frame are assigned different weight as a perspective normalization.

5- Find the SIFT descriptor for each motion edge pixel. Then, cluster the SIFT descriptors to 256 clusters. The centres of SIFT features are used as criteria for clustering them.
$F_{S I F T}=\operatorname{SIFT}(i, j)$
$(i, j) \in$ motion edge
$F_{\text {Cluster }}=$ Cluster $\left(F_{\text {SIFT }}\right)$
$(i, j) \in$ motion edge

Where $F_{\text {SIFT }}$ is the SIFT descriptors of the frame and $F_{\text {Cluster }}$ is the SIFT descriptors clustering. 
6- Divided the frames into cells (as a grid map) and count the number of people in each cell.

$F_{\text {Grid }}=\sum_{n} C_{n}$

Where $F_{\text {Grid }}$ is the grid map of each frame, $C$ is a cell in the grid map and $n$ is the number of cells in the grid map. Four cells configuration has been used in the proposed system which gives the best accuracies experimentally.

7- Use a quadratic programming (Interior-point-convex algorithm) to find the density of each cluster in each cell.

8- Integrate the densities of pixels over each cell to find the number of people in each cell.

$$
N_{\text {cell }}=\sum_{(i, j) \in B_{n}} P_{\text {density }}(i, j)
$$

Where $N_{\text {cell }}$ is the number of people in each cell and $P_{\text {density }}(i, j)$ is the density of each pixel that belongs to this cell.

9- The summation of the number of people in all cells represents the total number of people in the frame.

$$
N_{\text {total }}=\sum_{n} N_{\text {cell }}
$$

Where $N_{\text {total }}$ is the total number of people in a frame and $n$ is the number of cells. 


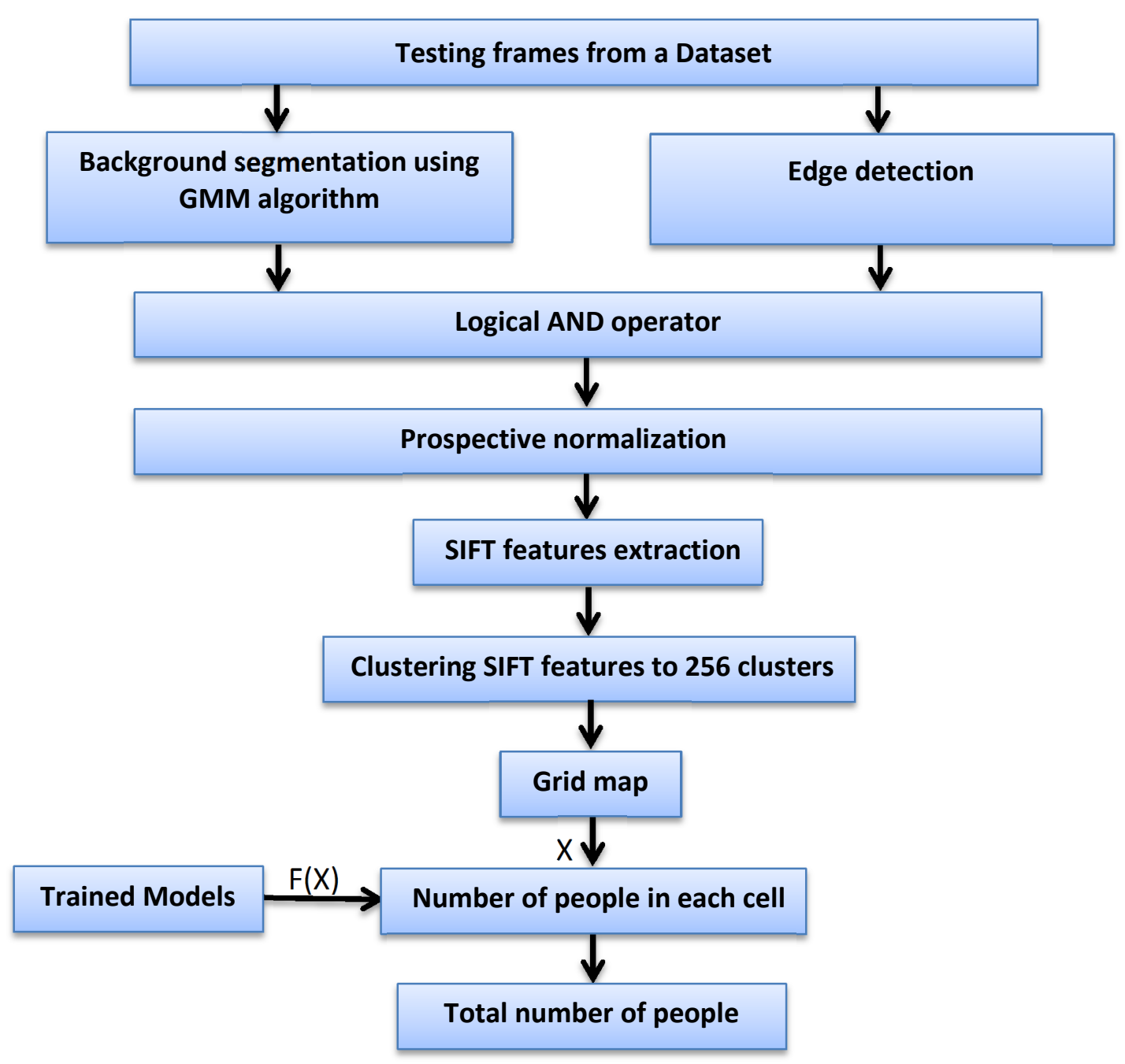

Figure 2: Flow diagram of the SIFT Features algorithm.

\subsection{Algorithm 2: SIFT-FAST features algorithm}

This algorithm uses two features; FAST and SIFT. This algorithm combines the following techniques to count the number of people; motion edges, grid map, SIFT \& FAST features and pixel-wise techniques. Edge pixels are used because their number is less than those of foreground pixels. The same approach as for SIFT feature algorithm described in Section 3.1 is used. However, FAST corner points are used to improve the accuracy due to the high correlation between the number of people and FAST corner points. The algorithm can also better adapt to high variations due to crowd behaviours, distribution and density. Figure 3 shows the flow diagram of the algorithm. Steps 1 to 5 are the same as for SIFT feature algorithm and descriptions from step 6 are as follows:

6- Find FAST points in each frame within the motion region.

$$
F_{F A S T}=F(i, j) \quad(i, j) \in \text { motion regions }
$$


Where $F_{F A S T}$ is the FAST corner points of a frame.

7- All pixels that are FAST corner points are assigned the value 257 so that quadratic programming can be used to find 257 density values instead of 256.

8- Divide the frame into cells (as a grid map) and the number of people in each cell is counted individually.

$F_{\text {Grid }}=\sum_{n} C_{n}$

Where $F_{\text {Grid }}$ is the grid map of the frames, $C$ is a cell in the grid map and $n$ is the number of cells in the grid map.

9- Use a quadratic programming (Interior-point-convex algorithm) to find the density value of each cluster.

10- Integrate the densities of pixels over each cell to find the number of people in each cell.

$$
N_{\text {cell }}=\sum_{(i, j) \in B_{n}} P_{\text {density }}(i, j)
$$

Where $N_{\text {cell }}$ is the number of people in each cell and $P_{\text {density }}(i, j)$ is the density of each pixel that belongs to the cell.

11- The summation of the number of people in all cells represents the total number of people in each frame.

$N_{\text {total }}=\sum_{n} N_{\text {cell }}$

Where $N_{\text {total }}$ is the total number of people in a frame, $n$ is the number of cells. 


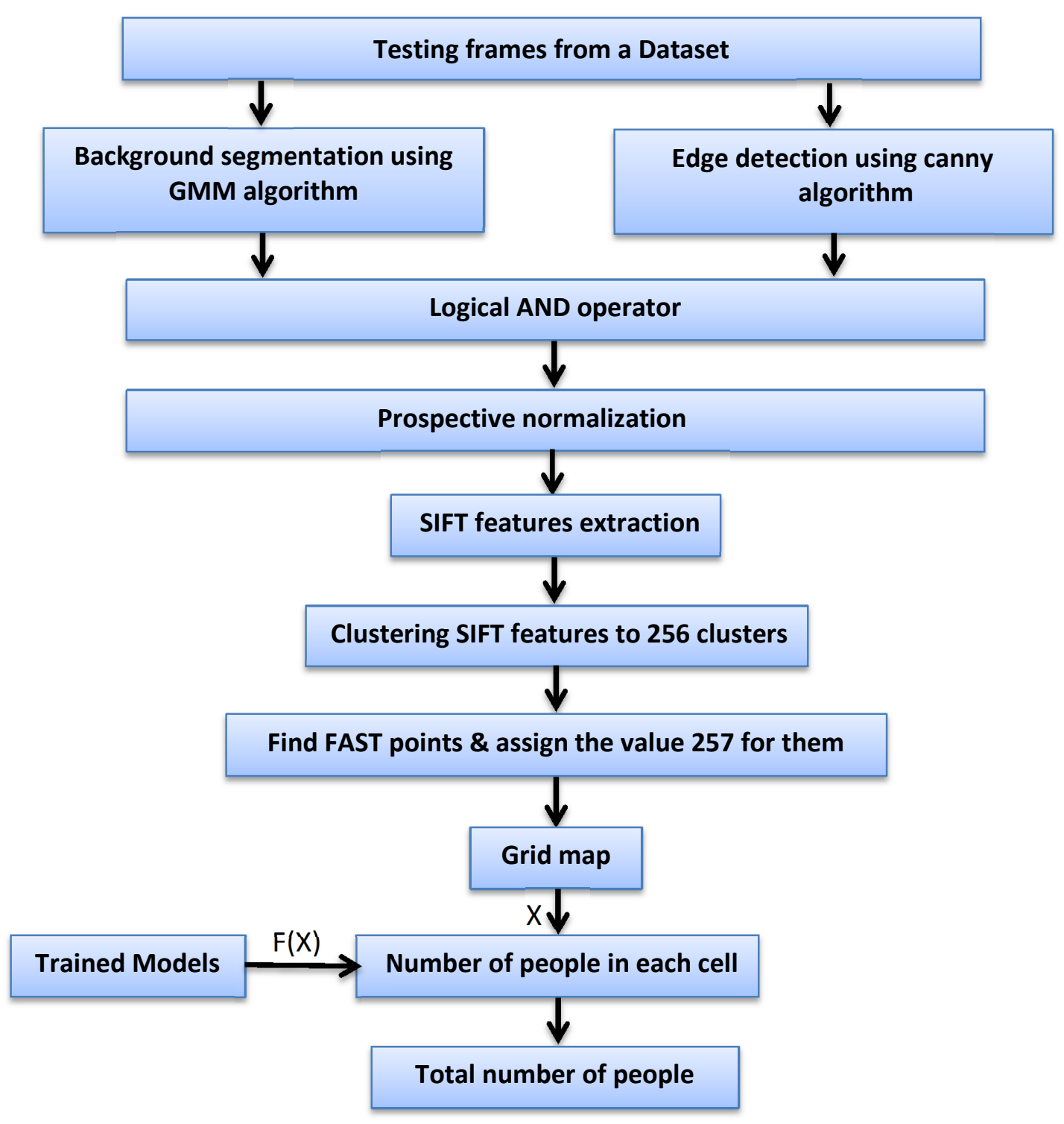

Figure 3: Flow diagram of the SIFT-FAST features algorithm.

\subsection{Fusion technique}

The fusion model is updated periodically using the results of all the algorithms. Each algorithm works independently to count the number of people and then they update the fusion model. Fusion is used to improve accuracy by determining the average error for each frame and to increase the confidence of the proposed system because the result of one algorithm is confirmed by that of another. This produces a cooperative paradigm and improves the confidence level in the results.

\subsection{Geometric correction}

At long distances, people appear smaller than those closer to the camera. Therefore, the extracted features of the same person at different locations in the scene are significantly different. 
Re-scaling the pixels of the frames is implemented by assigning different weights to solve this problem. Figure 4 shows the different sizes of the same pedestrian at different depths. Line $(a b)$ is the reference line so the pixel's weight on that line is 1 , the pixels of other lines are scaled and weighted using equation (18) [8];

$$
\text { weight }_{\text {line }}=\frac{h_{a b} w_{a b}}{h_{\text {line }} w_{\text {line }}}
$$

Where $h_{\text {line }}$ and $h_{a b}$ are the heights of a person at the line of interest and the height of the same person at the $(a b)$ line, respectively. $w_{\text {line }}$ and $w_{a b}$ are the width of the rectangle at the line of interest and at $(a b)$ line, respectively.
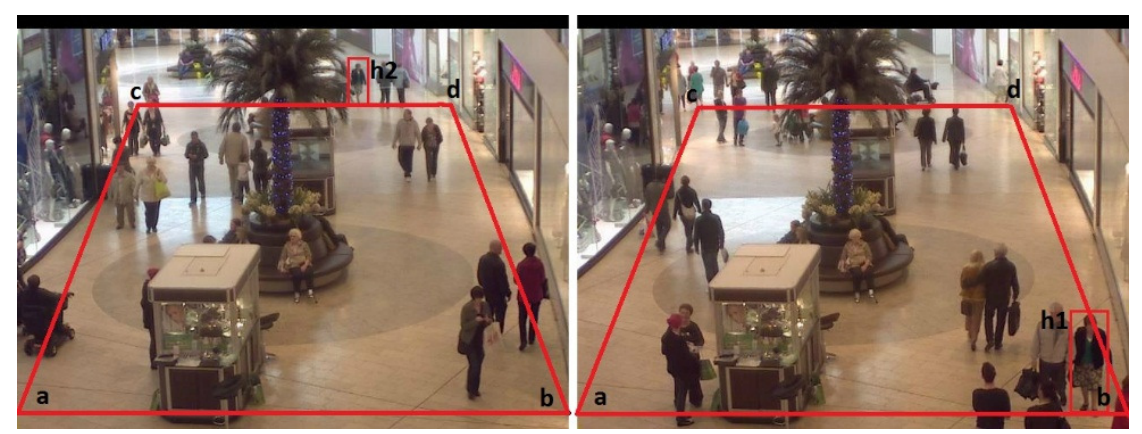

Figure 4: The change of size of the same person at different locations.

\subsection{Background segmentation}

Background segmentation is a process of extracting foreground information on a frame by frame basis. Background segmentation algorithms usually consist of three steps; background initialization, foreground detection and background maintenance [70]. In the background initialization, various techniques such as statistical, fuzzy and neuro-inspired techniques are used to build a background model. In foreground detection, a comparison is implemented between the current frame and the background model. Updating a background model according to changes in the environment is processed in the background maintenance step. Background segmentation methods can be classified into recursive and non-recursive algorithms [16]. In non-recursive algorithms, the background model is considered to be static and does not update, whereas in recursive algorithm, it is a dynamic and changes depending on the change of environment [16]. Figure 5 shows the general block diagram of background segmentation algorithms. 


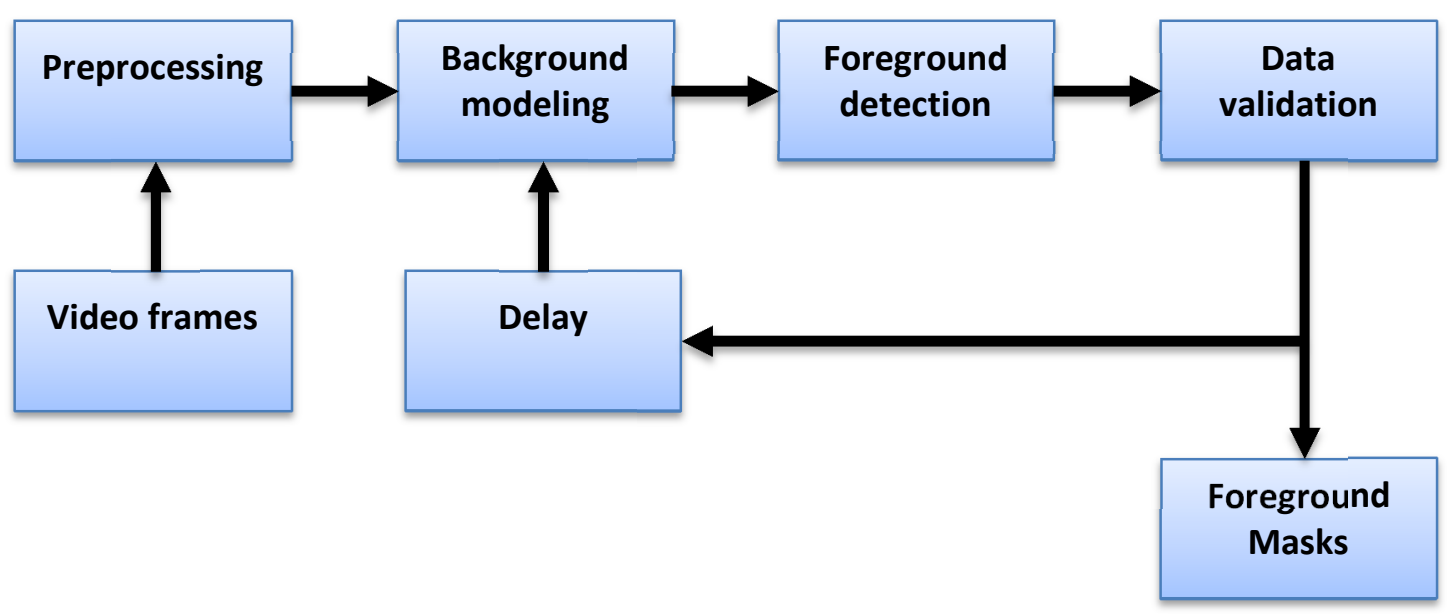

Figure 5: General block diagram of background segmentation algorithms.

GMM is one of the most widely used algorithms for background segmentation. This algorithm is a robust in light varying conditions and in environments with animated textures such as waves on the surface of water or trees being blown by wind [71]. Each pixel in a background model is formed using a mixture of Gaussian distributions (normally from three to five distributions) rather than one Gaussian distribution [71], [72].

$$
p\left(X_{t}\right)=\sum_{i=1}^{K} w_{i, t} * f\left(x_{t} \mid \mu_{i, t}, \Sigma_{i, t}\right)
$$

Where $K$ is the number of Gaussian distributions and $w_{i, t}$ is the weight of the $i^{\text {th }}$ distribution at time t. Each Gaussian distribution can be found using the probability density function;

$$
f(x \mid \mu, \Sigma)=\frac{1}{\sqrt{(2 \pi)^{n}|\Sigma|}} \exp \left(-\frac{1}{2}(x-\mu)^{T} \Sigma^{-1}(x-\mu)\right)
$$

Where $\mu$ is the mean and $\Sigma$ is the covariance matrix. The background model is updated using an adaptive filter;

$$
\mu_{t}=\alpha X_{t}+(1-\alpha) \mu_{t-1}
$$

Where;

- $\mu_{t}$ denotes the spatial mean of the pixels at time $\mathrm{t}$,

- $\mu_{t-1}$ denotes the previous spatial mean of the pixels at time $\mathrm{t}-1$,

- $\alpha$ is an empirical weight and

- $X_{t}$ is the current pixels values. 


\subsection{Edge detection}

They refer to the process of localising pixel intensity transitions [73]. There is a strong relationship between the complexity of crowds and the number of people because crowded environments tend to produce complex edges, while sparse environments tend to produce coarse edges [8]. Edges can be extracted using different algorithms such as Sobel, Canny, Prewitt, Roberts and Fuzzy logic algorithms [74], [75]. Canny edge detection is used in the proposed system. The following steps explain the procedure of canny edge algorithm [76]:

1- Smooth the image using a Gaussian filter to minimise noise.

$$
S(i, j)=G(i, j, \Sigma) * I(i, j)
$$

Where $G(i, j, \boldsymbol{\Sigma})$ is a Gaussian filter and $I(i, j)$ is a pixel.

2- Use derivative approximation by finite differences to find gradient magnitude and orientation. Firstly, partial derivatives $X(i, j)$ and $Y(i, j)$ is found by using the smoothed array $S(i, j)$ :

$$
\begin{aligned}
& X(i, j) \approx(S(i, j+1)-S(i, j)+S(i+1, j+1)-S(i+1, j)) / 2 \\
& Y(i, j) \approx(S(i, j)-S(i+1, j)+S(i, j+1)-S(i+1, j+1)) / 2
\end{aligned}
$$

The partial derivatives $X(i, j)$ and $Y(i, j)$ are then used to find the magnitude and orientation of the gradient:

$$
\begin{aligned}
& M(i, j)=\sqrt{X(i, j)^{2}+Y(i, j)^{2}} \\
& \theta(i, j)=\arctan (X(i, j), Y(i, j))
\end{aligned}
$$

3- Non-Maximal Suppression algorithm (NMS) is performed to thin out the edges. The edges are then detected using the double thresholding algorithm.

\subsection{Clustering}

Clustering is used in the proposed system to reduce the number of different descriptors (hundreds of thousands for $640 \times 480$ frame size) into a reasonable number of clusters (256 clusters in the SIFT features algorithm and 257 clusters in the SIFT-FAST features algorithm) that can be used with quadratic programming. K-means clustering is a method of vector quantisation and aims to partition $n$ observations into $k \leq n$ clusters such that each observation belongs to the cluster with the nearest mean [77]. In other words, it aims to find:

$$
\operatorname{argmin}_{S} \sum_{i=1}^{K} \sum_{X \in S_{i}}\left\|X-\mu_{i}\right\|^{2}
$$

Where $X$ is the observation, $S_{i}$ is the $i^{\text {th }}$ cluster and $\mu_{i}$ is the mean of cluster $S_{i}$. In the proposed system, the k-means algorithm is used to cluster the SIFT descriptors of the datasets frames and produce a 
codebook of 256 entries. The codebook is constructed using only the descriptors of the training frames and then the descriptors of the testing frames are clustered by the K-means algorithm and the codebook. The SIFT descriptor of each pixel is represented by one value between 1 and 256. A vector of length 256 is used to convert each pixel and quantise it by comparing them with the centroids in the codebook.

\section{RESULTS AND DISCUSSION}

\subsection{Benchmark datasets}

The pedestrian dataset from University of California, San Diego (UCSD) and the Mall datasets have been used to evaluate the proposed system [17], [18]. UCSD dataset has been widely used for testing and validating people counting methods [78]. Mall dataset is a newer and more comprehensive dataset to use in that it covers a different range of crowd densities, different activity patterns (static and moving crowds), collected under a large range of illumination conditions at different times of the day with more severe perspective distortion. Thus individual objects may exhibit larger variations in size and appearance at different depths of the scene [8]. The Mall dataset was introduced by Chen [18]. It has been collected inside a cluttered indoor and includes 2000 annotated frames. The two datasets have the same length (2000 frames) but they have different features in terms of the frame rate (fps), resolution, colour, location, shadows, reflections, crowd size and frame type [4], [62]. Table 1 shows the features of each dataset.

For the Mall and UCSD datasets, the datasets are partitioned into a training set, for learning the proposed system, and a test set, for validation. 100 frames from different locations of each dataset are allocated indivdually for training and 1900 frames for testing.

Table 1: The features of the benchmark datasets.

\begin{tabular}{|c|c|c|}
\hline & Mall dataset & UCSD dataset \\
\hline Year & 2012 & 2008 \\
\hline Length (frames) & 2000 & 2000 \\
\hline Frame rate (fps) & $<2$ & 10 \\
\hline Resolution & $640 \times 480$ & $238 \times 158$ \\
\hline Colour & RGB & Grey \\
\hline Location & Indoor & Outdoor \\
\hline Shadows & Yes & No \\
\hline Reflections & Yes & No \\
\hline Loitering & Yes & No \\
\hline Crowd size & $11-45$ & $13-53$ \\
\hline Frame type & .jpeg & .png \\
\hline
\end{tabular}

\subsection{Evaluation metrics}

Three metrics have been used as performance indicators for crowd counting; Mean deviation Error (MDE), mean absolute error (MAE) and mean squared error (MSE) [8]. The MDE is defined as; 


$$
M D E=\frac{1}{N} \sum_{n=1}^{N} \frac{\left|y_{n}-\hat{y}_{n}\right|}{y_{n}}
$$

The MAE is defined as;

$$
M A E=\frac{1}{N} \sum_{n=1}^{N}\left|y_{n}-\hat{y}_{n}\right|
$$

The MSE is given as;

$$
M S E=\frac{1}{N} \sum_{n=1}^{N}\left(y_{n}-\hat{y}_{n}\right)^{2}
$$

Where $N$ is the total number of the test frames, $y_{n}$ is the actual count, and $\hat{y}_{n}$ is the estimated count of $n_{t h}$ frames. MAE and MSE are indicative quantities of the error of the estimated crowd count but they contain no information about how crowded the environment is [8]. MDE takes into account the crowdedness and gives an indication of how good a measurement is relative to the actual count [79].

\subsection{Background segmentation, edge detection and motion edge extraction}

The GMM is used for background segmentation and the Canny edge algorithm is performed to extract the edges of the frames. The logical 'AND' is used to extract motion edge. Figures 6 and 7 show the results of the background segmentation, edge detection and motion edge extraction of two sample frames, one from the Mall dataset and the second from the UCSD dataset.

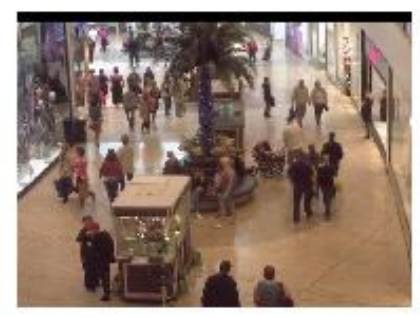

(a)

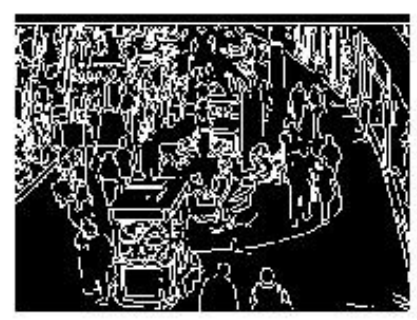

(c)

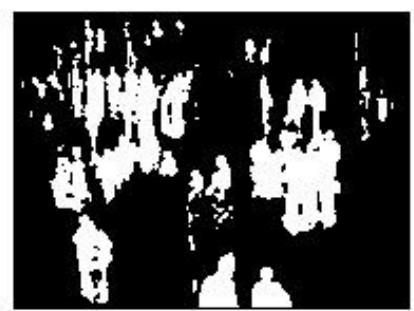

(b)

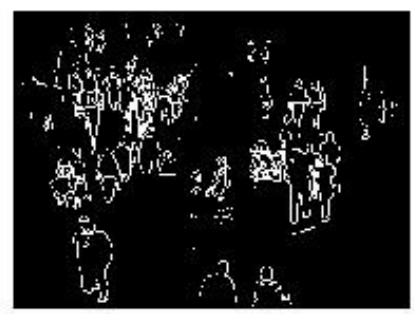

(d)

Figure 6: (a) An example of the Mall dataset form; (b) foreground, using GMM algorithm; (c) edge using Canny detector; (d) the motion edge, using logical 'AND'. 


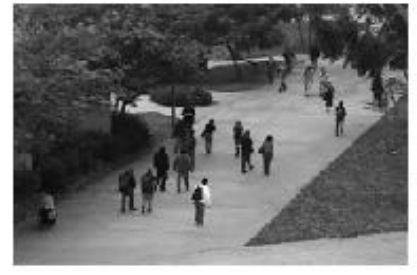

(a)

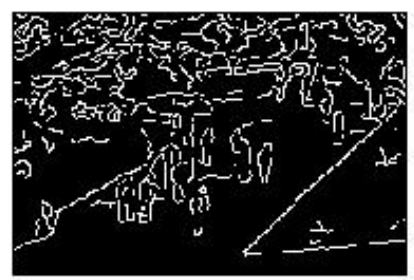

(c)

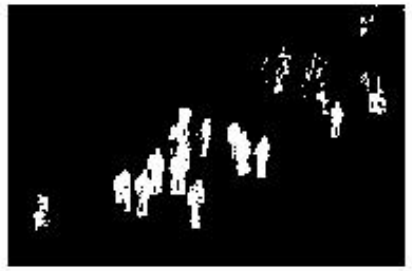

(b)

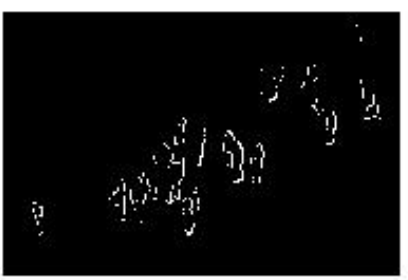

(d)

Figure 7: (a) An example of the UCSD dataset form; (b) foreground, using GMM algorithm; (c) edge using Canny detector; (d) the motion edge, using logical 'AND'.

\subsection{Performance evaluation of the proposed system using the Mall dataset}

As shown in Table 2, the mean deviation error (MDE) of the SIFT features algorithm is 0.099 and 0.094 for SIFT-FAST features algorithm. The results are compared with results presented by other researchers for the same dataset as a measure of accuracy of the proposed system. From the results, we can see that the accuracy of the SIFT-FAST features algorithm is slightly better than that of SIFT features algorithm. It shows that there is a reasonable improvement in the accuracies of the implemented algorithms when compared to those published by other researchers. Figure 8 shows the percentage of frames within the MDE distribution of the algorithms. Figure 9 shows the true count (TC) of people from sample frames of the Mall dataset, which is annotated by red dots. EC1 and EC2 represent the estimated number of people using SIFT and SIFT-FAST features algorithms, respectively.

The performance of crowd systems is measured using the accuracy (MAE, MSE and MDE) and practicality. Practicality is measured by the percentage of the training frames minimisation [43]. Crowd counting systems are practical if they are easy to deploy. In the real world, crowd systems are deployed in different environments which means they are individually trained for the location. Therefore, it is very important to reduce the number of the training frames required. The ground truth (the actual number of people) for each training frame is required when training crowd counting systems. Each environment needs several hundreds of frames (usually 400-800 training frames) for the training [50], [81]-[83], so the training process becomes time-consuming. 
The results of the proposed system have been compared with recent results from other researchers for the evaluation. The comparison with other methods based on the accuracy metrics (MAE, MSE and $\mathrm{MDE}$ ) is not enough to measure the performance for many reasons: firstly, pixel-wise optimisation based algorithms can be trained using a small number of frames in comparison to regression based algorithms [69]. The proposed system uses 100 frames for the training whilst the other state of the art methods use between 400 and 800 frames [50], [81]-[83]. In conclusion, the proposed system is more practical because the set-up time is faster by a factor of at least four (uses 4 times less training frames) compared to regression based algorithms which lead also to low set-up cost. Secondly, the lower number of training frames required in the training stage reduces the potential for error being introduced because manually annotation is an error-prone task. The accuracy of crowd counting systems are significantly affected by errors in the training stage. Thirdly, the proposed system is a multipurpose system because it can be used for crowd counting and also in people detection [32].

The comparison is only used to show that although the proposed system reduces the training error, speed, cost and can be used to develop more accurate people detection methods, its accuracy is, at least, comparable with the state of the art methods. None of the published results presented in Table 2 performs better than SIFT-FAST features algorithm based on the metrics used in this paper. In terms of MSE, only the algorithms presented in [18], [58] and [80] produced slightly better results but not in terms of MAE and MDE metrics. Finally, the MDE of the proposed system is less than the acceptable error (0.2) which is meeting the minimum accuracy requirements of system operators [62].

Table 2: Comparison for the Mall dataset results between the proposed system and the state of the art algorithms.

\begin{tabular}{|l|c|c|c|}
\hline \multirow{2}{*}{ Algorithm } & \multicolumn{3}{c|}{ Mall dataset } \\
\cline { 2 - 4 } Algorithm 1: SIFT Features Algorithm & MAE & MSE & MDE \\
\hline Algorithm 2: SIFT-FAST Features Algorithm & $\mathbf{3 . 0 8}$ & $\mathbf{1 6 . 3 1}$ & $\mathbf{0 . 0 9 9}$ \\
\hline Cumulative attribute based model (CA-RR) [81] & 3.43 & $\mathbf{1 4 . 6 4}$ & $\mathbf{0 . 0 9 4}$ \\
\hline $\begin{array}{l}\text { Squares Support Vector Machine Regression } \\
\text { (LSSVR) [81] }\end{array}$ & 3.51 & 17.70 & 0.105 \\
\hline Kernel Ridge Regression (KRR) [81] & 3.51 & 18.20 & 0.108 \\
\hline Random Forest Regression (RFR) [81] & 3.91 & 21.50 & 0.108 \\
\hline Gaussian Process Regression (GPR) [18], [81] & 3.72 & 20.1 & 0.121 \\
\hline Ridge regression (RR) [18], [81] & 3.59 & 19.00 & 0.110 \\
\hline Multi Output Ridge Regression (MORR) [18] & 3.15 & 15.70 & 0.099 \\
\hline Multiple Localised Regression (MLR) [18] & 3.90 & 23.90 & 0.119 \\
\hline Weighted Ridge Regression (WRR) [82] & 3.44 & 18.00 & 0.105 \\
\hline Random Projection Forest (RPF) [58] & 3.22 & 15.50 & - \\
\hline $\begin{array}{l}\text { Cost-sensitive Sparse Linear Regression (CS- } \\
\text { SLR) [80] }\end{array}$ & 3.23 & 15.77 & 0.104 \\
\hline
\end{tabular}




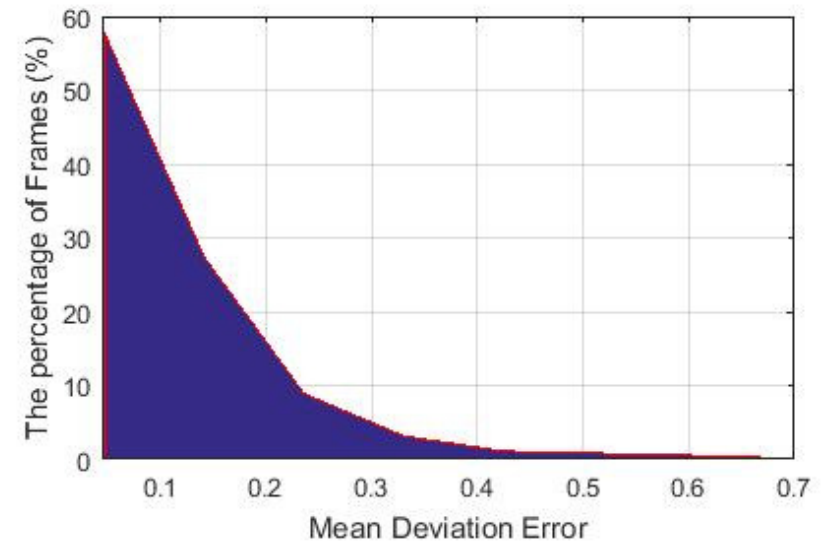

(a)

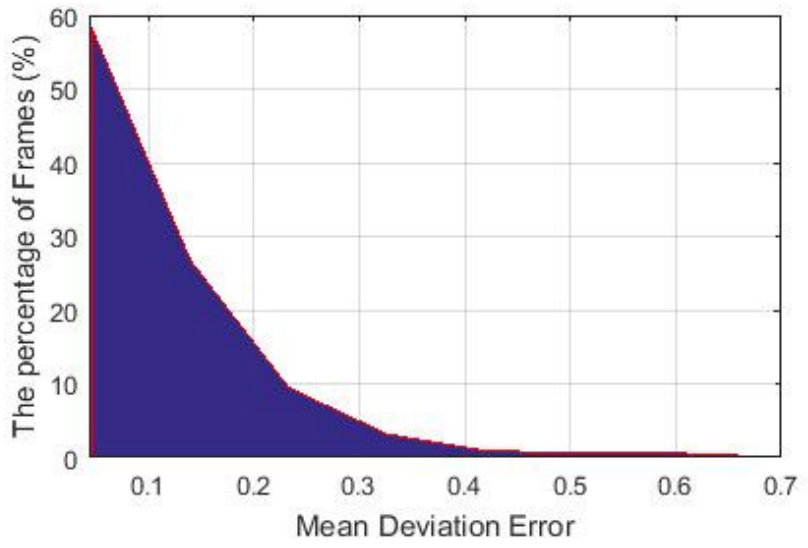

(b)

Figure 8: (a) The MDE of SIFT algorithm (b) the MDE of SIFT-FAST algorithm.

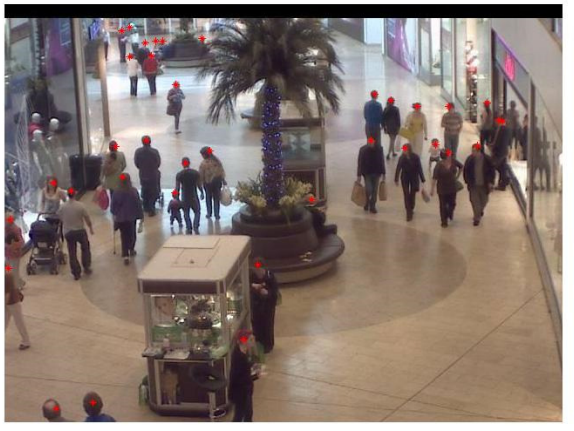

(a) $\mathrm{TC}=36, \mathrm{EC} 1=38, \mathrm{EC} 2=37$

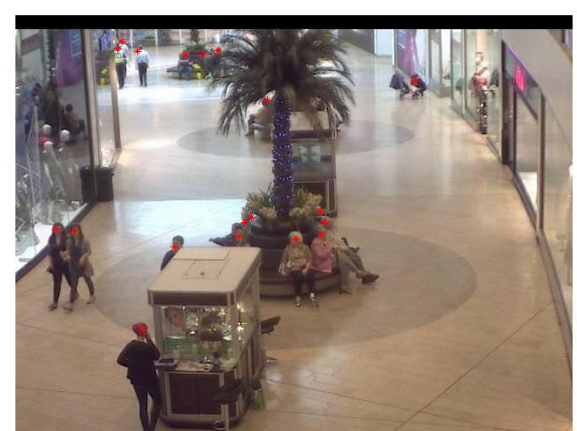

(c) $\mathrm{TC}=19, \mathrm{EC} 1=20, \mathrm{EC} 2=20$

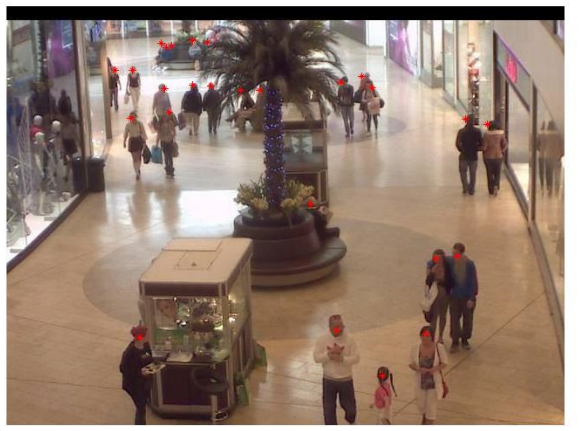

(b) $\mathrm{TC}=26, \mathrm{EC} 1=29, \mathrm{EC} 2=25$

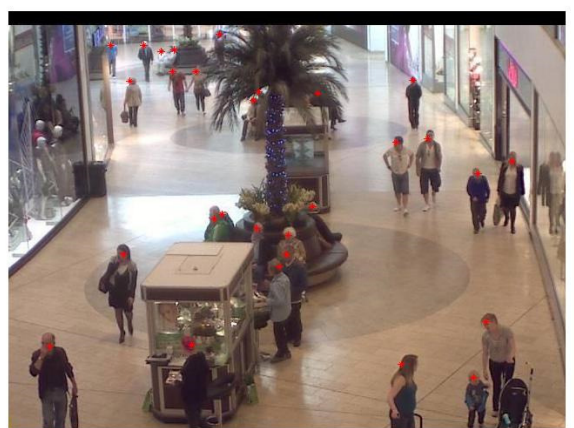

(d) $\mathrm{TC}=29, \mathrm{EC} 1=32, \mathrm{EC} 2=28$

Figure 9: Examples of the true count (TC) \& the estimated count of people using SIFT (EC1) and SIFT-FAST (EC2) algorithms.

\subsection{Performance evaluation of the proposed system using the UCSD dataset}

The UCSD dataset represents people moving in two directions along a walkway. As shown in Table 3, the MDE of SIFT features algorithm is 0.066 and 0.064 for SIFT-FAST features algorithm. From 
the results, it can be seen that the accuracy of SIFT-FAST features algorithm is better than that of SIFT features algorithm. Figure 10 shows the percentage of frames within the MDE distribution of the algorithms. Figure 11 shows the true count (TC) of people from sample frames of the UCSD dataset, which is annotated by red dots. In general, the accuracies of the proposed system with the UCSD dataset are better than the results from the Mall dataset. The potential justification is that the Mall dataset is more complicated in terms of shadows, reflections and crowd size [4], [62]. In addition, the Mall dataset is collected with more severe perspective distortion than the UCSD dataset. As is the case with the MDE from the Mall dataset, the MDE of this dataset is significantly lower than the acceptable error (0.2) which is meeting the minimum accuracy requirements of system operators [62]. Results of both datasets show that their average accuracies for each dataset are almost similar but their accuracies at frame level are different. The difference of estimation between the SIFT and SIFT-FAST algorithms for each frame is usually between 0 and 4. EC1 and EC2 at each frame are correlative because both algorithms use almost the same approach. However, FAST corner points are used with SIFT-FAST features algorithm to improve the accuracy due to the high correlation between the number of people and FAST corner points. SIFT-FAST features algorithm gives the best results compared to all published results presented in Table 3. Only results presented in [80] gives a comparable results to the SIFT features algorithgm.

Table 3: Comparison for the UCSD dataset results between the proposed system and the state of the art algorithms.

\begin{tabular}{|l|c|c|c|}
\hline \multicolumn{2}{|c|}{ Algorithm } & \multicolumn{2}{c|}{ UCSD dataset } \\
\cline { 2 - 4 } & MAE & MSE & MDE \\
\hline Algorithm 1: SIFT Features Algorithm & $\mathbf{1 . 8 2}$ & $\mathbf{5 . 2 4}$ & $\mathbf{0 . 0 6 6}$ \\
\hline Algorithm 2: SIFT-FAST Features Algorithm & $\mathbf{1 . 7 6}$ & $\mathbf{4 . 9 3}$ & $\mathbf{0 . 0 6 4}$ \\
\hline $\begin{array}{l}\text { Improved Iterative Scaling -Label Distribution Learning } \\
\text { (IIS-LDL) [83] }\end{array}$ & 2.08 & 7.25 & 0.098 \\
\hline Kernel Ridge Regression (KRR) [83] & 2.16 & 7.45 & 0.107 \\
\hline Random Forest Regression (RFR) [83] & 2.42 & 8.47 & 0.116 \\
\hline Gaussian Process Regression (GPR) [82], [83] & 2.24 & 7.97 & 0.112 \\
\hline Ridge Regression (RR) [82], [83] & 2.25 & 7.82 & 0.110 \\
\hline Multi Output Ridge Regression (MORR) [83] & 2.29 & 8.08 & 0.109 \\
\hline Cumulative attribute based model (CA-RR) [81], [83] & 2.07 & 6.86 & 0.102 \\
\hline Weighted Ridge Regression (WRR) [82] & 2.05 & 6.75 & 0.102 \\
\hline $\begin{array}{l}\text { Linear regression (LR), Partial Least Squares } \\
\text { Regression (PLSR), KRR, LSSVR, GPR and RFR [8] }\end{array}$ & $>2.02$ & $>6.67$ & $>0.100$ \\
\hline Random Projection Forest (RPF) [58] & 1.90 & 6.01 & - \\
\hline Cost-sensitive Sparse Linear Regression (CS-SLR) [80] & 1.83 & 5.04 & 0.079 \\
\hline Moving SIFT algorithm [84] & 3.26 & - & 0.180 \\
\hline
\end{tabular}




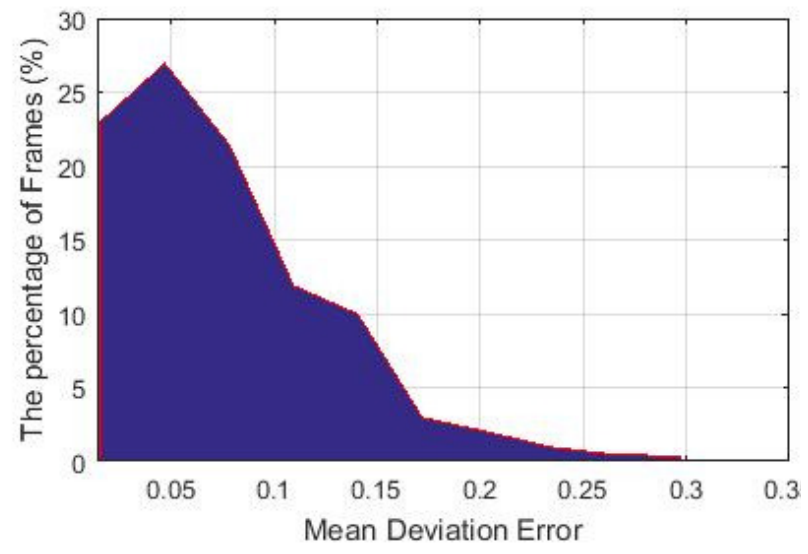

(a)

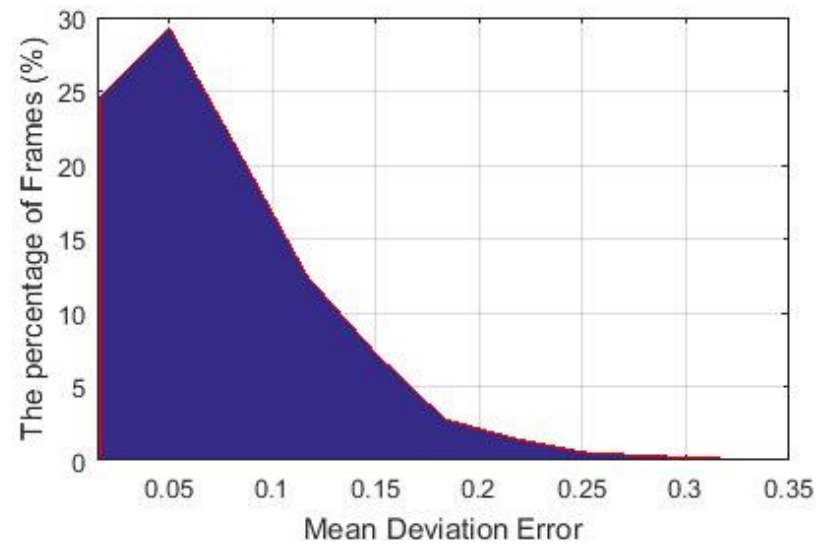

(b)

Figure 10: (a) MDE of SIFT algorithm (b) MDE of SIFT-FAST algorithm.

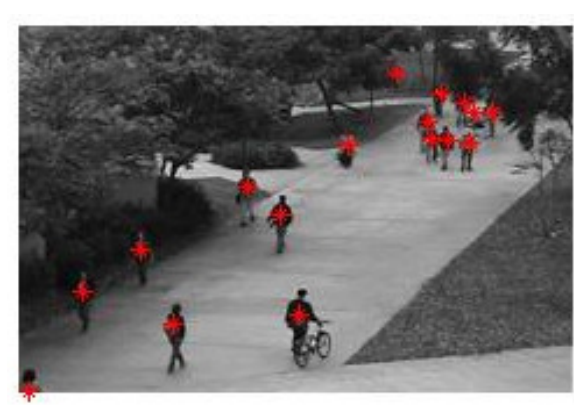

(a) $\mathrm{TC}=18, \mathrm{EC} 1=19, \mathrm{EC} 2=18$

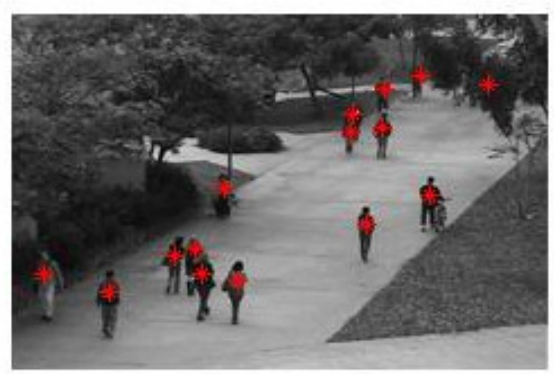

(c) $\mathrm{TC}=15, \mathrm{EC} 1=15, \mathrm{EC} 2=17$

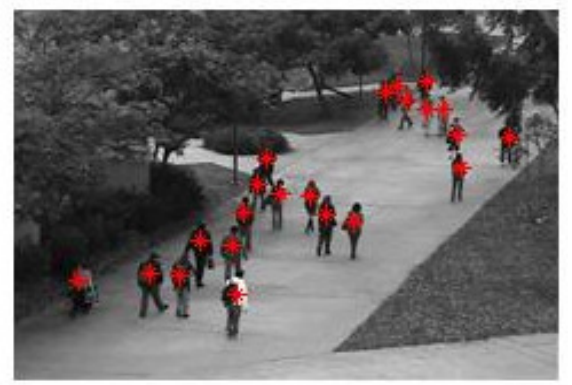

(b) $\mathrm{TC}=23, \mathrm{EC} 1=22, \mathrm{EC} 2=24$

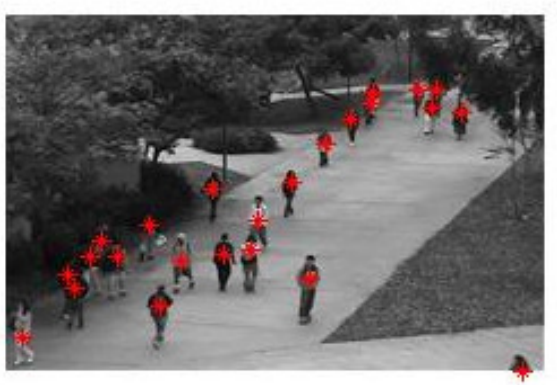

(d) $\mathrm{TC}=23, \mathrm{EC} 1=21, \mathrm{EC} 2=22$

Figure 11: Examples of the true count (TC) \& the estimated count of people using SIFT (EC1) and SIFT-FAST (EC2) algorithms. 


\subsection{Performance evaluation of the proposed system in sparse and crowded scenarios}

To evaluate the proposed system with sparse and crowded scenarios, the test set of the Mall dataset is split the same as in [8] into a sparse set which includes all the frames with ground truth (number of people), less than or equal to 30 , and crowded set which includes all the frames with ground truth values greater than 30 . The test set of the UCSD dataset is also split the same as in [8] into a sparse set which includes all the frames that their ground truth is less than or equal to 23 , and crowded set which includes all the frames that their ground truth is greater than 23 .

To ensure that the proposed system is practical and robust, the training set was not been split because the technical definition of the boundary that separates the sparse and crowded frames is not clear [56]. In addition, partitioning the training set into two sets would required two training stages. The test sets are processed by the proposed system jointly and then the results are analysed by splitting them into sparse and crowded sets. In conclusion, the split between sparse and crowded scenarios have mainly been carried out by identifying which frames could be classified into each of the categories. No differential training of the system has been carried out. Tables 4 and 5 show the results of both algorithms with sparse and crowded scenarios. The MDE of both algorithms in the sparse scenarios is higher than the MDE crowded scenarios. The proposed system is more applicable for high density crowds and this can be seen from the achieved good results in crowded scenarios. This opens the door for using the proposed system in a high crowded environments. Figure 12 and 13 show the percentages of frames within the MDE distribution for the sparse and crowded scenarios based on the Mall and UCSD datasets, respectively.

Table 4: System performance with sparse and crowded scenarios (Mall dataset).

\begin{tabular}{|l|c|c|c|c|c|c|}
\hline \multirow{2}{*}{ Algorithm } & \multicolumn{3}{|c|}{ Sparse scenario } & \multicolumn{3}{c|}{ Crowded scenario } \\
\cline { 2 - 7 } & MAE & MSE & MDE & MAE & MSE & MDE \\
\hline SIFT features Algorithm & 3.20 & 18.39 & 0.126 & 2.96 & 14.27 & 0.081 \\
\hline SIFT-FAST features Algorithm & 3.15 & 17.21 & 0.124 & 2.73 & 12.11 & 0.075 \\
\hline
\end{tabular}

Table 5: System performance with sparse and crowded scenarios (UCSD dataset).

\begin{tabular}{|l|c|c|c|c|c|c|}
\hline \multirow{2}{*}{ Algorithm } & \multicolumn{3}{|c|}{ Sparse scenario } & \multicolumn{3}{c|}{ Crowded scenario } \\
\cline { 2 - 7 } & MAE & MSE & MDE & MAE & MSE & MDE \\
\hline SIFT features Algorithm & 1.67 & 4.41 & 0.093 & 1.93 & 5.84 & 0.055 \\
\hline SIFT-FAST features Algorithm & 1.65 & 4.29 & 0.084 & 1.84 & 5.41 & 0.056 \\
\hline
\end{tabular}




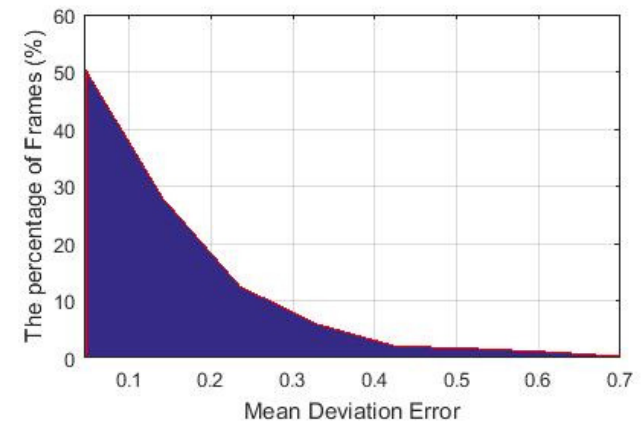

(a)

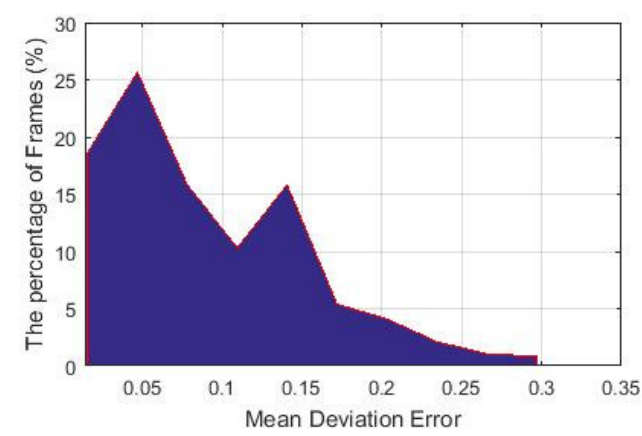

(c)

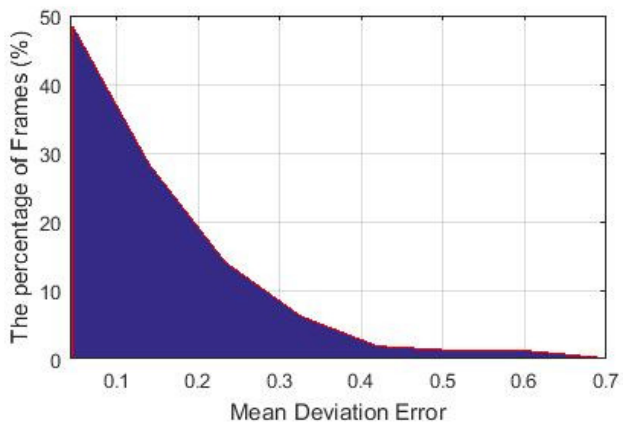

(b)

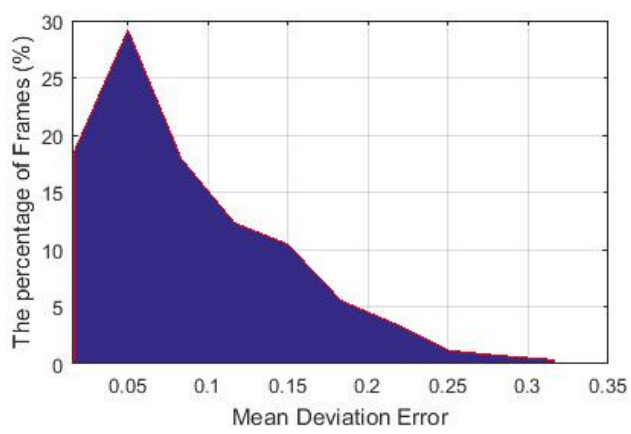

(d)

Figure 12: System performance with sparse scenarios. (a) and (b) are the MDE of the of SIFT algorithm and SIFTFAST algorithm on Mall dataset, respectively; (c) and (d) are the MDE of the of SIFT algorithm and SIFT-FAST algorithm on UCSD dataset, respectively.

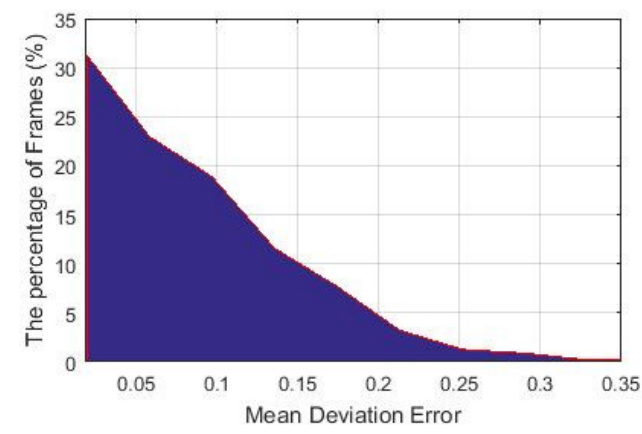

(a)

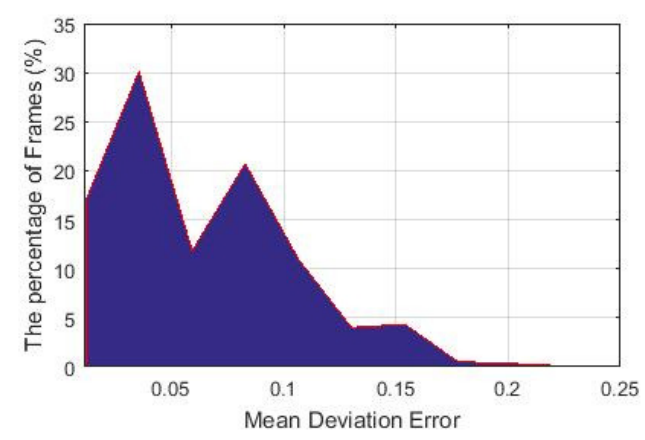

(c)

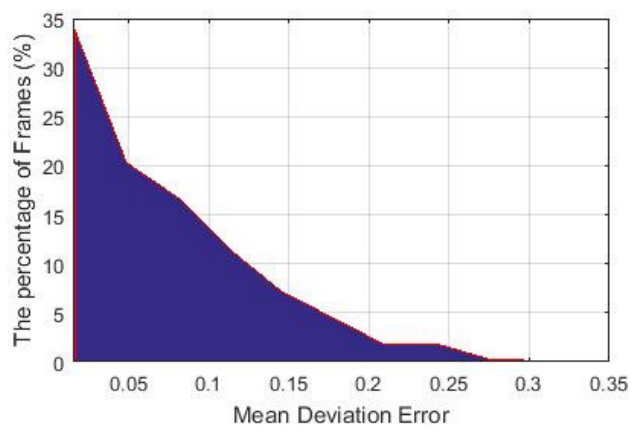

(b)

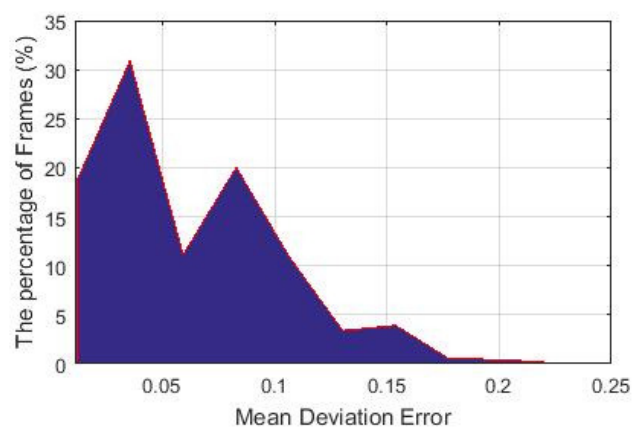

(d)

Figure 13: : System performance with crowded scenarios. (a) and (b) are the MDE of the of SIFT algorithm and SIFT-FAST algorithm on Mall dataset, respectively; (c) and (d) are the MDE of the of SIFT algorithm and SIFTFAST algorithm on UCSD dataset, respectively. 


\section{CONCLUSIONS}

CCTV cameras are already widely used, the objective of the research presented in this paper was to develop a system that can be incorporated with existing CCTV cameras to provide the number of people in a given space. Two algorithms have been proposed and implemented using a novel combination of four techniques; motion edges, grid map, SIFT \& FAST features and pixel-wise techniques. The use of edge pixels for which their number is small compared to foreground pixels significantly reduces the run time of the algorithms. SIFT and FAST features have been chosen due to their high correlation with the number of people. In addition, a grid map approach has been proposed and used to allow similar clusters in different cells to be assigned different densities depending on their location in the frame. This is used to improve the adaption of the proposed algorithms to high variations in crowd behaviours, distributions, lighting and densities.

The UCSD and Mall datasets have been used to evaluate the proposed system. The results have shown that the proposed algorithms achieve good results in heavily occluded environment with perspective distortions. Comparisons with the low-level features regression based methods published in literature show that the proposed algorithms improve the accuracies based on MDE, MSE and MAE metrics (less than 0.1, 16.5 and 3.1, respectively, for the Mall dataset and less than 0.07, 5.5 and 1.9, respectively, for UCSD dataset). The proposed system is more practical than low-level features regression based methods because it can be trained with a lower number of frames so it is relatively easy to deploy. In addition, it reduces the training error, speed, cost and, opens the door to developing more accurate people detection methods. The proposed algorithms can also be used to estimate crowd densities at specific locations in a scene. This shows significant promise as it can be used to detect localised abnormalities in applications such crowd control, evacuation planning and product displays. Comparison of the proposed system in sparse and crowded scenarios shows that it performs better in crowded environments.

\section{References:}

[1] C. Norris, M. Mccahill, and D. Wood, "Editorial . The Growth of CCTV : a global perspective on the international diffusion of video surveillance in publicly accessible space .," Surveill. Soc., vol. 2, no. 2/3, pp. 110-135, 2004.

[2] X. Xing, K. Wang, and Z. Lv, "Fusion of Gait and Facial Features using Coupled Projections for People Identification at a Distance,” Signal Process. Lett. IEEE, vol. 22, no. 12, pp. 2349-2353, 2015.

[3] J. Wang, W. Fu, J. Liu, H. Lu, and S. Member, "Spatiotemporal Group Context for Pedestrian Counting," vol. 24, no. 9, pp. 1620-1630, 2014.

[4] S. A. M. Saleh, S. A. Suandi, and H. Ibrahim, "Recent survey on crowd density estimation and counting for visual surveillance,” Eng. Appl. Artif. Intell., vol. 41, pp. 103-114, 2015.

[5] Y. L. Hou and G. K. H. Pang, "People counting and human detection in a challenging situation," IEEE Trans. Syst. Man, Cybern. Part ASystems Humans, vol. 41, no. 1, pp. 24-33, 2011.

[6] X. Hu, H. Zheng, Y. Chen, and L. Chen, "Dense crowd counting based on perspective weight model using a fisheye camera," Opt. - Int. J. Light Electron Opt., vol. 126, no. 1, pp. 123-130, 2015. 
[7] D. A. Ryan, “Crowd Monitoring Using Computer Vision,” Queensland University of Technology, 2013.

[8] C. Loy, K. Chen, S. Gong, and T. Xiang, Crowd counting and profiling: Methodology and evaluation. 2013.

[9] T. Bouwmans, F. El Baf, and B. Vachon, "Background Modeling using Mixture of Gaussians for Foreground Detection - A Survey," Recent Patents Comput. Sci., vol. 1, no. 3, pp. 219-237, 2008.

[10] ShopperTrak, "ShopperTrak Solutions," 2013. [Online]. Available: www . shoppertrak.com / products. [Accessed: 23-Mar-2015].

[11] A. Technology, “Our customers," 2013. [Online]. Available: www.peoplecounting.co.uk/our-customers. [Accessed: 23-Mar-2015].

[12] Biodata Ltd, "Use CCTV to Count People," 2013. [Online]. Available: www.videoturnstile.com. [Accessed: 23-Mar-2015].

[13] DILAX Intelcom, "Public Transport," 2015. [Online]. Available: www.dilax.net/electronic-people-counterpassenger-counter-customer-counter/passenger-counting-systems-for-public-transportation. [Accessed: 23Mar-2015].

[14] K. Hashimoto, K. Morinaka, N. Yoshiike, C. Kawaguchi, and S. Matsueda, "People count system using multisensing application,” Proc. Int. Solid State Sensors Actuators Conf. (Transducers '97), vol. 2, pp. 1291-1294, 1997.

[15] M. Wang, "Data Assimilation for Agent-Based Simulation of Smart Environment," 2014.

[16] A. Adegboye, G. Hancke, and G. H. Jr, "Single-pixel approach for fast people counting and direction estimation," Satnac.Org.Za.

[17] A. B. Chan, Z. S. J. Liang, and N. Vasconcelos, "Privacy preserving crowd monitoring: Counting people without people models or tracking," 26th IEEE Conf. Comput. Vis. Pattern Recognition, CVPR, 2008.

[18] K. Chen, C. C. Loy, S. Gong, and T. Xiang, "Feature Mining for Localised Crowd Counting," Procedings Br. Mach. Vis. Conf. 2012, p. 21.1-21.11, 2012.

[19] J. Tu, C. Zhang, and P. Hao, "Robust real-time attention-based head-shoulder detection for video surveillance," Image Process., pp. 3340-3344, 2013.

[20] N. Dalal and B. Triggs, "Histograms of Oriented Gradients for Human Detection," CVPR '05 Proc. 2005 IEEE Comput. Soc. Conf. Comput. Vis. Pattern Recognit. - Vol. 1, pp. 886-893, 2005.

[21] P. Dollar, S. Belongie, and P. Perona, "The Fastest Pedestrian Detector in the West," Procedings Br. Mach. Vis. Conf. 2010, p. 68.1-68.11, 2010.

[22] O. Sidla, Y. Lypetskyy, N. Brändle, and S. Seer, "Pedestrian detection and tracking for counting applications in crowded situations," Proc. - IEEE Int. Conf. Video Signal Based Surveill. 2006, AVSS 2006, 2006.

[23] O. Tuzel, F. Porikli, and P. Meer, "Pedestrian Detection via Classification on Riemannian Manifolds," IEEE Trans. Pattern Anal. Mach. Intell., vol. 30, no. 10, 2008.

[24] B. Leibe, E. Seemann, and B. Schiele, "Pedestrian detection in crowded scenes," Comput. Vis. Pattern Recognition, 2005. CVPR 2005. IEEE Comput. Soc. Conf., vol. 1, pp. 878-885, 2005.

[25] P. F. Felzenszwalb, R. B. Girshick, D. McAllester, and D. Ramanan, "Object Detection with Discriminative Trained Part Based Models,” IEEE Trans. Pattern Anal. Mach. Intell., vol. 32, no. 9, pp. 1627-1645, 2010.

[26] S. Lin, J. Chen, and H. Chao, "Estimation of Number of People in Crowded Scenes Using Perspective Transformation," IEEE Trans. Syst. Man. Cybern., vol. 31, no. 6, pp. 645-654, 2001.

[27] B. Wu and R. Nevatia, "Detection and tracking of multiple, partially occluded humans by Bayesian combination of edgelet based part detectors," Int. J. Comput. Vis., vol. 75, no. 2, pp. 247-266, 2007.

[28] M. Harville, "Stereo person tracking with adaptive plan-view statistical templates," Proc. ECCV Work. Stat. Methods Video Process., pp. 67-72, 2002.

[29] J. Li, L. Huang, and C. Liu, "Robust people counting in video surveillance: Dataset and system," $20118 t h$ IEEE Int. Conf. Adv. Video Signal Based Surveillance, AVSS 2011, pp. 54-59, 2011.

[30] W. Ge and R. T. Collins, "Marked point processes for crowd counting," 2009 IEEE Comput. Soc. Conf. Comput. Vis. Pattern Recognit. Work. CVPR Work. 2009, pp. 2913-2920, 2009.

[31] H. Ma, C. Zeng, and C. X. Ling, "A Reliable People Counting System via Multiple Cameras,” ACM Trans. Intell. Syst. Technol., vol. 3, no. 2, pp. 1-22, 2012. 
[32] M. Rodriguez, E. N. Superieure, I. Laptev, J. Sivic, and J.-Y. Audibert, "Density-aware person detection and tracking in crowds," Comput. Vis. (ICCV),IEEE, pp. 2423-2430, 2011.

[33] G. J. Brostow and R. Cipolla, "Unsupervised Bayesian Detection of Independent Motion in Crowds," Comput. Vis. Pattern Recognition, 2006.

[34] V. Rabaud and S. Belongie, "Counting crowded moving objects," Proc. IEEE Comput. Soc. Conf. Comput. Vis. Pattern Recognit., vol. 1, pp. 705-711, 2006.

[35] I. S. Topkaya, H. Erdogan, and F. Porikli, “Counting People by Clustering Person Detector Outputs," pp. 313318, 2014.

[36] A. M. Cheriyadat, B. L. Bhaduri, and R. J. Radke, "Detecting multiple moving objects in crowded environments with coherent motion regions," 2008 IEEE Comput. Soc. Conf. Comput. Vis. Pattern Recognit. Work. CVPR Work., 2008.

[37] D. Merad, K. E. Aziz, and N. Thome, "Fast people counting using head detection from skeleton graph," Proc. - IEEE Int. Conf. Adv. Video Signal Based Surveillance, AVSS 2010, pp. 233-240, 2010.

[38] D. Berndt and J. Clifford, "Using dynamic time warping to find patterns in time series," Work. Knowl. Knowl. Discov. Databases, vol. 398, pp. 359-370, 1994.

[39] G. Antonini and J. P. Thiran, "Trajectories clustering in ICA space an application to automatic counting of pedestrians in video sequences," No. EPFL-CONF-87110.IEEE, 2004.

[40] M. Wang and X. Wang, "Automatic adaptation of a generic pedestrian detector to a specific traffic scene," Proc. IEEE Comput. Soc. Conf. Comput. Vis. Pattern Recognit., pp. 3401-3408, 2011.

[41] R. Shbib, S. Zhou, D. Ndzi, and K. Al-kadhimi, "Distributed Monitoring System Based On Weighted Data Fusing Model,” Am. J. Soc. Issues Humanit., vol. 3, no. March, pp. 53-62, 2013.

[42] T. W. S. Chow, J. Y. F. Yam, and S. Y. Cho, "Fast training algorithm for feedforward neural networks: Application to crowd estimation at underground stations," Artif. Intell. Eng., vol. 13, no. 3, pp. 301-307, 1999.

[43] D. Ryan, S. Denman, C. Fookes, and S. Sridharan, "Crowd counting using multiple local features," DICTA 2009 - Digit. Image Comput. Tech. Appl., pp. 81-88, 2009.

[44] S.-Y. Cho, T. Chow, and C. Leung, "A neural-based crowd estimation by hybrid global learning algorithm," IEEE Trans. Syst. Man, Cybern. Part B Cybern., vol. 29, no. 4, pp. 535-541, 1999.

[45] T. W. Cho, S. Y., \& Chow, "A fast neural learning vision system for crowd estimation at underground stations platform,” Neural Process. Lett., vol. 10(2), pp. 111-120, 1999.

[46] J. Zhang, B. Tan, F. Sha, and L. He, "Predicting pedestrian counts in crowded scenes with rich and highdimensional features," IEEE Trans. Intell. Transp. Syst., vol. 12, no. 4, pp. 1037-1046, 2011.

[47] A. B. Chan and N. Vasconcelos, "Counting people with low-level features and bayesian regression," IEEE Trans. Image Process., vol. 21, no. 4, pp. 2160-2177, 2012.

[48] A. B. Chan and N. Vasconcelos, "Bayesian poisson regression for crowd counting," Proc. IEEE Int. Conf. Comput. Vis., no. Iccv, pp. 545-551, 2009.

[49] A. Chan, M. Morrow, and N. Vasconcelos, "Analysis of Crowded Scenes using Holistic Properties," 9th Int. Symp. PETS, no. Pets, 2009.

[50] D. Conte, P. Foggia, G. Percannella, F. Tufano, and M. Vento, "A method for counting people in crowded scenes,” Proc. - IEEE Int. Conf. Adv. Video Signal Based Surveillance, AVSS 2010, pp. 225-232, 2010.

[51] L. Xiaohua, S. Lansun, and L. Huanqin, "Estimation of Crowd Density Based on Wavelet and Support Vector Machine," Trans. Inst. Meas. Control, vol. 28, no. 3, pp. 299-308, 2006.

[52] R. Ma, L. Li, W. Huang, and Q. Tian, "On pixel count based crowd density estimation for visual surveillance," IEEE Conf. Cybern. Intell. Syst. 2004., vol. 1, pp. 1-3, 2004.

[53] A. Davies, J. H. Yin, and S. Velastin, "Crowd monitoring using image processing,” no. February, 1995.

[54] H. Fradi and J. L. Dugelay, "Low level crowd analysis using frame-wise normalized feature for people counting," WIFS 2012 - Proc. 2012 IEEE Int. Work. Inf. Forensics Secur., pp. 246-251, 2012.

[55] Y. Hu, H. Chang, F. Nian, Y. Wang, and T. Li, "Dense crowd counting from still images with convolutional neural networks,” J. Vis. Commun. Image Represent., vol. 38, pp. 530-539, 2016.

[56] Z. Q. H. Al-Zaydi, D. L. Ndzi, Y. Yang, and M. L. Kamarudin, “An adaptive people counting system with dynamic features selection and occlusion handling,” J. Vis. Commun. Image Represent., vol. 39, pp. 218-225, 
2016.

[57] A. Hafeezallah and S. Abu-Bakar, "Crowd counting using statistical features based on curvelet frame change detection," Multimed. Tools Appl., 2016.

[58] B. Xu and G. Qiu, "Crowd Density Estimation based on Rich Features and Random Projection Forest," IEEE Winter Appl. Comput. Vis., 2016.

[59] A. S. Rao, J. Gubbi, S. Marusic, and M. Palaniswami, "Estimation of crowd density by clustering motion cues," Vis. Comput., vol. 31, no. 11, pp. 1533-1552, 2015.

[60] N. C. Tang, Y. Lin, M. Weng, and H. M. Liao, "Cross-Camera Knowledge Transfer for Multiview People Counting," IEEE Trans. Image Process., vol. 24, no. 1, pp. 80-93, 2015.

[61] L. Gao, Y. Wang, X. Ye, and J. Wang, "Crowd Pedestrian Counting Considering Network Flow Constraints in Videos," arXiv Prepr., 2016.

[62] D. Ryan, S. Denman, S. Sridharan, and C. Fookes, "An evaluation of crowd counting methods, features and regression models," Comput. Vis. Image Underst., vol. 130, pp. 1-17, 2015.

[63] D. Kong, D. Gray, and H. Tao, "Counting Pedestrians in Crowds Using Viewpoint Invariant Training," Procedings Br. Mach. Vis. Conf. 2005, p. 63.1-63.10, 2005.

[64] D. Kong, D. Gray, and H. Tao, "A viewpoint invariant approach for crowd counting," Proc. - Int. Conf. Pattern Recognit., vol. 3, pp. 1187-1190, 2006.

[65] H. Çelik, A. Hanjalić, and E. a. Hendriks, "Towards a robust solution to people counting," Proc. - Int. Conf. Image Process. ICIP, pp. 2401-2404, 2006.

[66] P. Kilambi, E. Ribnick, A. J. Joshi, O. Masoud, and N. Papanikolopoulos, "Estimating pedestrian counts in groups," Comput. Vis. Image Underst., vol. 110, no. 1, pp. 43-59, 2008.

[67] D. Conte, P. Foggia, G. Percannella, F. Tufano, and M. Vento, "Counting moving people in videos by salient points detection," Proc. - Int. Conf. Pattern Recognit., pp. 1743-1746, 2010.

[68] S. W. Jeong, C. Y., Choi, S., \& Han, "A method for counting moving and stationary people by interest point classification," Image Process., pp. 4545-4548, 2013.

[69] V. Lempitsky and A. Zisserman, "Learning To Count Objects in Images," pp. 1-9, 2010.

[70] A. Sobral and A. Vacavant, "A comprehensive review of background subtraction algorithms evaluated with synthetic and real videos," Comput. Vis. Image Underst., vol. 122, pp. 4-21, 2014.

[71] A. O. Adegboye, "Single pixel robust approach for background subtraction for fast people-counting and direction estimation," University of Pretoria, 2013.

[72] Y. Benezeth, P.-M. Jodoin, B. Emile, H. Laurent, and C. Rosenberger, "Comparative study of background subtraction algorithms," J. Electron. Imaging, vol. 19, no. 3, p. 33003, 2010.

[73] D. Ryan, S. Denman, C. Fookes, and S. Sridharan, "Scene invariant multi camera crowd counting," Pattern Recognit. Lett., vol. 44, pp. 98-112, 2014.

[74] G. Kaur and I. S. Virk, "Edge Detection through Fuzzy System using Type I Format," vol. 102, no. 13, pp. 24-27, 2014.

[75] N. S. Joshi and N. S. Choubey, "Comparison of Traditional Approach for Edge Detection with Soft Computing Approach," vol. 96, no. 11, pp. 17-23, 2014.

[76] G. T. Shrivakshan and C. Chandrasekar, "A Comparison of various Edge Detection Techniques used in Image Processing,” Int. J. Comput. Sci. Issues, vol. 9, no. 5, pp. 269-276, 2012.

[77] T. Bottesch, K. Markus, M. Kaechele, and U. N. I. Ulm, "Speeding up k -means by approximating Euclidean distances via block vectors," Proc. 33rd Int. Conf. Mach. Learn., vol. 48, pp. 2578-2586, 2016.

[78] C. Zhang, H. Li, and X. Wang, "Cross-scene Crowd Counting via Deep Convolutional Neural Networks," Proc. IEEE Conf. Comput. Vis. Pattern Recognit., 2015.

[79] A. Hafeezallah and S. Abu-Bakar, "Crowd counting using statistical features based on curvelet frame change detection," Multimed. Tools Appl., 2016.

[80] X. Huang, Y. Zou, and Y. Wang, "Cost-sensitive sparse linear regression for crowd counting with imbalanced training data," in IEEE International Conference on Multimedia and Expo (ICME), 2016.

[81] K. Chen, S. Gong, T. Xiang, and C. C. Loy, "Cumulative attribute space for age and crowd density 
estimation," Proc. IEEE Comput. Soc. Conf. Comput. Vis. Pattern Recognit., no. 2, pp. 2467-2474, 2013.

[82] K. Chen and J.-K. Kamarainen, "Learning to Count with Back-Propagated Information," Pattern Recognit., pp. 4672-4677, 2014.

[83] Z. Zhang, M. Wang, and X. Geng, "Crowd counting in public video surveillance by label distribution learning," Neurocomputing, vol. 166, pp. 151-163, 2015.

[84] D. Conte, P. Foggia, G. Percannella, and M. Vento, "Counting moving persons in crowded scenes," Mach. Vis. Appl., vol. 24, no. 5, pp. 1029-1042, 2013. 